\title{
An Extension of Earley's Algorithm for S-Attributed Grammars
}

\author{
Nelson Correa \\ Department of Electrical Engineering \\ Universidad de los Andes \\ Apartado Aéreo 4976, Bogotá, D.E., Colombia \\ bitnet : NCORREA at ANDESCOL
}

\begin{abstract}
Aturibute grammars are an clegant formalization of the augmented context-frec grammars characteristic of most current natural language systems. This paper presents an cxtension of Earley's algorithm to Knuth's attributc grammars, considering the case of $S$-attributed grammars. For this case, we study the conditions on the underlying base grammar under which the extended algorithm may be guarantecd to tcrminate. Finile partitioning of attributc domains is proposed to guarantec the termination of the algorithm, without the necd for any restrictions on the context-frec base.
\end{abstract}

\section{Introduction}

Earley's (1970) algorithm is a gencral algorithm for context-free languages, widely used in natural language processing (King, 1983; Shicber, 1985) and syntactic pattern recognition ( $\mathrm{Fu}, 1982$ ), where the full generative power of context-free grammar is required. The original algorithm and its common implementations, however, assume the atomic symbols of context-free grammars, thus limiting its applicability to systems with attributed symbols, or attribute grammars (Knuth, 1968).

Attribute grammar is an elegant formalization of the augmented context-frec grammars characteristic of most current NLP systems. It is more general than members of the family of unification-based grammar formalisms (Kay, 1985; Shicber, 1986), mainly in that it allows and cncourages the use of simpler attribution functions than unification for the definition of attribute values, and hence can lead to computationally. efficient grammatical definitions, while maintaining the advantages of a well-understood declarative formalism. Attribute grammar has been used in the past by the author to define computational models of Chomsky's Government-binding theory, from which practical parsing programs werc developed (Corrca, 1987a).

Many systcms based on Earley's algorithm have a clear division between the phases of syntactic and semantic analysis. Ycllin (1988), for instance, uses a syntactic analysis phase in which Earlcy's algorithm builds a factored parse tree (FPT) for the given input, which is then followed by up to two phases of semantic analysis, during which the FPT is attributed and cvaluated. Watt (1980) and Jones and Madsen (1980) propose a close intcraction between syntactic and scmantic analysis in the form of "attribute-directed" parsing. However, their particular realization of the technique is severely restricted for NLP applications, since it uses a detcrministic onc-path (LR) algorithm, applicablc only to scmantically unambiguous grammars.

Percira and Warren (1983) and Shicber (1985) present versions of Earlcy's algorithm for unification grammars, in which unification is the sole opcration responsible for attribute evaluation. Howcver, given the high computational cost of unification, important differences between attribute and unification grammars in their respective attribution domains and functions (Correa, forthcoming), and the more general nature of attribute grammars in this regard, it is of interest to investigate the extension of Earley's algorithm directly to the main subclasses of attribute grammar.

The paper is organized as follows: Section 2 presents prcliminary clements, including a definition of attribute grammar and Earlcy's algorithm. Section 3 presents the extension of the algorithm for $\mathrm{S}$-attributed grammars. In Section 4, we consider the conditions on the underlying grammar under which the extended algorithm may be guarantced to terminate for cach input. For the $S$-attributed case we show that the algorithm terminates if the grammar has no cycles or, equivalently, if it is finitcly ambiguous. However, finite partitioning of attribute domains may be used to guarantec the tcrmination of the algorithm, without the need for restrictions on the context-frec base. Finally, a conclusion and note on implementation are given.

\section{Notation and Preliminaries}

We follow the usual notation and terminology for grammars and languages. A language is a sct of surings over a finite set T of symbols. A grammar is a formal device for specifying which strings are in the set. In particular, a context-free grammar is a cuadruple 
$(N, T, P, S)$, where $N$ is a finite set of string categorics; $\mathrm{T}$ a finite set of tcrminal symbols; $\mathrm{P}$ a finitc set of productions or rewriting rules of the form $X \rightarrow \sigma$, $X \in N, \sigma \in(N \cup T)^{*}$; and $S$ a distinguished symbol of $N$. A binary relation $\Rightarrow$ of derivation between strings over the vocalulary NUT of the grammar is defined such that $\alpha X \beta \Rightarrow \alpha \sigma \beta$ iff $X \rightarrow \sigma$ is a production of $P$; now, $\Rightarrow *$ may be defined as the reflexive and transitive closure of $\Rightarrow$. The language generated by the grammar, noted $L(G)$, is the sct of strings $\omega \in T^{*}$, such that $S \Rightarrow{ }^{*} \omega$.

An altribute grammar is defined upon a context-frec grammar $G=(N, T, P, S)$, by associating each symbol $\mathrm{X} \in \mathrm{N} \cup \mathrm{T}$ with a finite set $\mathrm{A}(\mathrm{X})$ of attributes, and a type or domain $\operatorname{dom}(a)$ for each attribute $a$ thus defined (Knuth, 1968). Each attribute $a$ of X, noted X.a, takes values over its domain and represents a specific, possibly context-sensitive properly of the symbol.

Attribute values are defined by attribution rules of the form $X_{i} \cdot a \leftarrow f\left(X_{j} \cdot b, \ldots, X_{k} \cdot c\right)$, associated with each production $\mathrm{p}=\mathrm{X}_{\mathbf{0}} \rightarrow \mathrm{X}_{1} \ldots \mathrm{X}_{\mathrm{n}}$ in the grammar, $0 \leq \mathrm{i}, \mathrm{j}, \mathrm{k} \leq \mathrm{n}$. Here, $f$ is an applicativc cxpression (function) whose value depends on the values of attribute occurrences associatcd with symbols in the production. Each time $p$ applies in a derivation, the attribution rule defines the valuc of the attribute occurrence $X . a$ as a function of the occurrences $X_{j} . b, \ldots, X_{k} . c$, associated with other symbols in $p$. We let $\mathrm{R}(\mathrm{p})$ denote the packet of attribution rules associated with $p$. The grammar may also definc attribute conditions of the form $\mathrm{B}\left(\mathrm{X}_{\mathrm{i}} \cdot \mathrm{a}, \ldots, \mathrm{X}_{\mathrm{k}} \cdot \mathrm{b}\right), 0 \leq \mathrm{i}, \mathrm{k} \leq \mathrm{n}$, where $B$ is a Boolcan predicate on the values of attribute ocurrences in $p$. This condition must be satisficd in any derivation requiring the application of $p$, and thus contributes to the notion of grammaticality in the language generated by the grammar. We Ict B(p) denote the packet of attribute conditions associatcd with $p$.

The above remarks are summarized as follows: An attribute grammar is a cuadruple $A G=(G, A, R, B)$, where

i. $G=(N, T, P, S)$ is a context-frec grammar;

ii. $A=\bigcup_{X \in N \cup T} A(X)$ is a finite set of attributes;

iii. $R=\bigcup_{p \in P^{R}(p)}$ is a finite set of attribution rules, as above; and

iv. $B=\bigcup_{p \in P} B(p)$ is a finite set of attribute conditions, as above.

The base grammar $G$ assigns a derivation tree $\tau$ to cach sentence in $L(G)$. The tree is annotated at each node labelled $X$ with the set $A(X)$ of attributes associated with $X$; cach attribute $a \in A(X)$ defines an attribute occurrence $X . a$ at node $X$. If the grammar is well defincd (Knuth, 1968), it is possible to evaluate each attribute occurrence on the tree, and we say that $\tau$ is correctly attributed iff all attributc conditions yicld 'true.' The language generated by the aturibute grammar, $L(A G)$, is now the subset of $L(G)$ whose members have at least one correctly attributed trec.

It is possible to classify the attributes in AG according to the manner in which their values are defined. We say an attribute X.a is synthesized if its valuc depends only on attributes of daughters of $\mathrm{X}$; it is inherited if its value depends on attributes associated with the parent or sistcrs of $X$. We say the grammar is $S$-altributed if it contains only synthesized attributes. A more gencral and practically important class of $L$-attributed grammars is obtained if we allow attributes of both kinds, but such that each inherited attribute depends only on inherited attributes of the parent, or attributes of the sisters to its left (Bochmann, 1976).

Earlcy's algorithm is a recognizer for CFGs which uscs top-down prediction in combination with bottom-up parsing actions. Given an input string $x_{1}, \ldots, x_{n}$ it builds a state set $S_{i}$ at each position $i$ of the string, $0 \leq i \leq n+1$. Each slate in $S_{i}$ is of the form $\langle A \rightarrow \alpha \cdot \beta, f, \delta>$, where $A \rightarrow \alpha \cdot \beta$ is a dolted-production, $f$ an index to the position in the input string where this instance of the production began to be recognized $(0 \leq f$ $\leq i)$, and $\delta$ a string of $k$ symbols of lookahead $(k \geq 0)$. To begin, all state sets are initialized to empty and the initial state $\langle\phi \rightarrow \cdot S \perp, 0, \perp \mathbf{k}\rangle$ is put into $S_{0}$; here $\perp$ is the end-of-input marker. States are processed in order according to the position of their "dot" following three actions, Predictor, Completer, and Scanner, while maintaining the following invariant:

Statc $<A \rightarrow \alpha \cdot \beta, f, \delta>$ is in $S_{i}$ iff the following derivations are valid:

$\mathrm{S} \Rightarrow^{*} \sigma \mathrm{Av} ; \sigma \Rightarrow^{*} \times \mathrm{x} \ldots \mathrm{x}_{\mathrm{f}}$; and $\alpha \Rightarrow^{*} \mathrm{x}_{\mathrm{f}+1} \ldots \mathrm{x}_{\mathrm{i}}$.

Since the number of possible states is finite the algorithm terminatcs. The input string is accepted if $\left.S_{n+1}=\left\{<\phi \rightarrow S \perp \cdot, 0, \perp^{k}\right\rangle\right\}$. The correctness of this acceptance condition is a conscquence of the invariant.

\section{Extension to S-attributed Grammars}

The chicf element of the extension of the algorithm is a change in the representation of the states in Earley's original algorithm to attributed representations. Now, each dotted production $A \rightarrow \alpha \bullet \beta$ in a state consists of symbols attributed according to the grammar. For each category symbol $A$ in the base grammar, we define the attributed symbol $A\left[A . a_{1}, \ldots, A . a_{n}\right]$, where $A$ is the catcgory and $A . a_{i}, 1 \leq i \leq n$, an attribute occurrence of $A$.

The extended algorithm, in addition to syntactically recognizing the input string, evaluates the attribution associalcd with each of its possible derivations. In particular, for each derivation of the attributed final state 
$<\phi \rightarrow S\left[S . a_{1}, \ldots, S . a_{n}\right] \perp \cdot, 0, \perp^{k}>$, where $S$ is the start symbol of the grammar and $S . a_{1}, \ldots, S . a_{n}$ the attribute occurrences of $S$ associated with that state, the algorithm cvaluates the corresponding attributc occurrences. For an S-attributed grammar, this is achicved by the following modification of Earley's algorithm, in its Completer step:

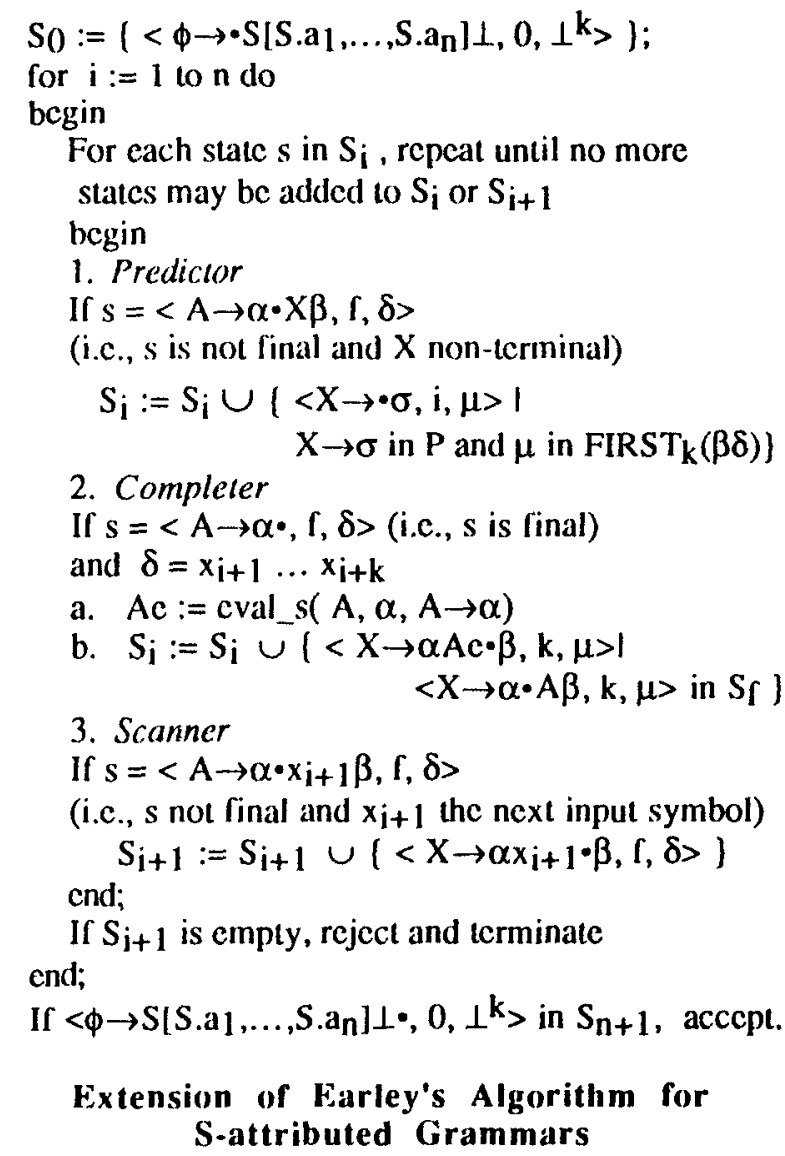

The states in the algorithm are attributed as indicated above. For example, the symbol $A$ in the state $\langle A \rightarrow \alpha \cdot, f, \delta>$ input to the Completer could be shown more explicitly as $A=A\left[A . a_{1}, \ldots, A . a_{n}\right]$. As the state cnters the completer, the attribute occurrences $A$.ai of $A$ are unevaluated; however, since the grammar is $S$-attributed it is casy to show that the attribute occurrences on the right-hand side $\alpha$ of the production have already becn evaluated. Hence, cvaluation of the attributc occurrences of $A$ reduces to application of the attribution associated with the production $A \rightarrow \alpha$, according to the attribute values in $\alpha$. This is done by the function eval_s $A, \alpha, A \rightarrow \alpha)$, which returns the attributed symbol $A c$, identical to $A$, except that its attribute occurrences have becn cvaluated, as required.

The last state set gencrated by the algorithm contains final states of the form $\langle\phi \rightarrow S[\ldots] \perp \cdot, 0, \perp k$, in which the attributed start symbol $S[\ldots]$ is already cvaluated. Here the extended algorithm differs form
Earley's; whereas the original algorithm gencrates at most onc final state, regardless of the ambiguity of the underlying grammar, the extended algorithm may generate scveral instances of this statc, if the grammar is ambiguous. Each instance of the final state corresponds to a different derivation of the initial symbol, lcading to a different cvaluation of the symbol's attributcs.

\section{Finite Partitioning of Attribute Domains}

The last remark in the extension of section 3 shows a defect of the Extended Algorithm: It may not terminate in the general case. For the S-attributed case, however, this may happen only if the underlying grammar is infinitcly ambiguous or, equivalently, if it has cycles or derivations of the form $A \Rightarrow^{+} A$, for some $A \in N$.

Consider, for example, the following grammar, which "measures" the Iength of each derivation of the sole string ' $a$ ' it generates:

$$
\begin{array}{ll} 
& \text { aluribution: } \\
S \rightarrow A & \text { S.v } \leftarrow A . v \\
A \rightarrow A & A_{0} \cdot v \leftarrow A_{1} \cdot v+1 \\
A \rightarrow a & \text { A.v } \leftarrow 1
\end{array}
$$

Given the input string ' $a$ ', the algorithm defines three attributted state sets:

$$
\begin{aligned}
& S_{0}=\left\{\left\langle\phi \rightarrow \cdot S[v] \perp, 0, \perp^{k}\right\rangle,\left\langle S[v] \rightarrow \cdot A[v], 0, \perp^{k}\right\rangle,\right. \\
& \left.\left\langle A[v] \rightarrow \cdot a, 0, \perp^{k}\right\rangle,\left\langle A[v] \rightarrow \bullet A[v], 0, \perp^{k}\right\rangle\right] \\
& S_{1}=\left\{\left\langle A \rightarrow a^{\bullet}, 0, \perp^{k}\right\rangle\right. \text {, } \\
& \left\langle S[v] \rightarrow A[1] \cdot, 0, \perp^{k}>,\left\langle A[v] \rightarrow A[1] \cdot, 0, \perp^{k}>\right.\text {, }\right. \\
& \left\langle\phi \rightarrow S[1] \cdot \perp, 0, \perp^{k}\right\rangle \\
& \left\langle S[v] \rightarrow A \cdot[2], 0, \perp^{k}\right\rangle,\left\langle A[v] \rightarrow A[2] \cdot, 0, \perp^{k}\right\rangle \text {, } \\
& \text { ad infinitum ...) } \\
& S_{2}=\left(\left\langle\phi \rightarrow S[1] \perp \cdot, 0, \perp^{k}\right\rangle\right. \text {, } \\
& <\phi \rightarrow S[2] \perp \cdot, 0, \perp k^{k} \text {, } \\
& \text { ad infinitum ...) }
\end{aligned}
$$

Since $S_{1}$ is infinite, the algorithm docs not tcrminate.

Cyclic grammars play an important role in most recent linguistic theorics, including Government-binding (GB), Lexical-Functional Grammar (LFG) and GPSG (cf. Bcrwick, 1988; Correa, 1987b; Kornai and Pullum, 1990). These have in common that they have shifted from rule-based descriptions of languagc, to declarative or principle-based descriptions, in which the role of phrase structure rulcs or principles is relatively minor. Thus, to make the extension of the algorithm uscful for natural language applications it becomes necessary to ensure its termination, in spitc of cyclic bases. 
The termination of the Extended Algorithm may be guarantecd whilc maintaining its full gencrality, through a finite partition on the attribute domains associated with cach cyclic symbol in the grammar. For each such domain dom $(a)$, the partition defines a finite collection of equivalence classes on attribute values. Now, before adding a ncw state $<A \rightarrow \alpha \cdot \beta, f, \delta>$ to a statc set $S_{i}$, wc tcst for equivalence (according to the defincd partitions) rather than equality to some previously added state; if the new state is cquivalent to some other, it is not added. It is casy to show that the number of attributed dotted items in the grammar, and hence the size of the state sets, is now finite. This number is in fact identical to that of Earlcy's algorithm, except for a constant multiplicative factor, dependent on the grammar and the size of the partitions selected for attribute domains. Since the sizc of the state sets possible with finitc partitioning is now finite, the algorithm always tcrminates.

After establishing a correspondence between attribute and unification grammar (UG), we may sce that the technique of "restriction" used by Shicber (1985) in his extended algorithm is related to finite partitioning on attribute domains, in fact a particular case which takes advantage of the more structured attribute domains of UG. For attribute grammar, given that the domains involved are more general (c.g., the integers), finite partitioning is the required device.

\section{Conclusions and Implementation Status}

This paper presented and extension of Earley's algorithm to $S$-altributed grammars. Combining on-line scmantic evaluation with the exccution of syntactic actions, the algorithm is an effective realization of attributc-directed parsing, as proposed by Watt (1980) and Jones and Madsen (1980). Although the algorithm is a recognizer, it computes the scmantic values associated with each derivation of the input string, and hence need not be extended to compute trec representations. In attribute grammars with conditions on productions, the values of attributes already evaluated may be used to guide the parsing process, reducing the number of states that may be generated by the algorithm.

The extension of the algorithm has becn written in " $\mathrm{C}$ ", using an efficient " $\mathrm{C}$ " implementation of Earlcy's original algorithm (Chamorro and Correa, 1990), and is currently being tested on small grammars. The extended algorithm will be the kcrnel of ANDES-1, a programming environment for attribute grammars, intended for natural language applications.

\section{References}

Berwick, Robert. 1988. "Principle-based Parsing". MIT A.I. Mcmo 972, reviscd. MIT, Cambridgc, MA.

Bochmann, Grcgor. 1976. "Scmantic Evaluation from Left to Right." Communications of the $A C M$, Vol. 19, No. 2, p. 55-62.

Chamorro, Miriam, and N. Correa. 1990. "An Efficicnt

'C' Implementation of Earley's Algorithm" - in Spanish. CIFI, Universidad de los Andes, Bogotá, Colombia.

Correa, Nelson. 1987a. "An Altribute Grammar Implementation of Government-binding Theory." Proceedings of the 25th Annual Meeting of the $A C L$, Stanford University, Stanford, CA.

Correa, Nelson. 1987b. "Empty Catcgorics, Chain Binding, and Parsing." Parsing Seminar; MIT Working Papers of the Lexicon Project, MIT, Cambridge, MA. Correa, Nelson. Forthcoming. Attribute and Unification Grammar: A Revicw of Formalisms and Comparision. CIFI, Universidad de los Andes.

Earley, Jay. 1970. "An Efficient Context-free Parsing Algorithm." Communications of the $A C M$, Vol, 13, No. 2, p. 94-102.

Fu, K.S. 1982. Syntactic Pattern Recognition. Academic Press, New York.

Jones, Neil D., and M. Madsen. 1980. "Attributc Influcnced LR Parsing." In N. D. Joncs, ed., Semantics-directed Compiler Generation, LNCS 94. Springer-Vcrlag, Ncw York.

Kay, Martin. 1985. Parsing in Functional Unification Grammar. In D. Dowty, L. Karttuncn, and A. Zwicky, eds., Natural Language Parsing , Cambridge University Press, Cambridge, England.

King, Margaret, ed. 1983. Natural Language Parsing . Academic Press, New York.

Knuth, Donald. 1968. "Semantics of Context-free Languages." Mathematical Systems Theory, Vol. 2, No. 2, p. 127-145.

Kornai, Andras, and G. Pullum. 1990. "The X-bar Theory of Phrase Structure." Language, Vol. 66.

Pereira, Fernando, and D. H. Warren. 1983. "Parsing as Deduction." Proceedings of the 21.st Annual Meeting of the ACL, MIT, Cambridge, MA.

Shicber, Stuart. 1985. "Using Restriction to Extend Parsing Algorithms for Complex-Fcature-Based Formalisms." Proceedings of the 23rd Annual Meeting of the $A C L$, University of Chicago, Chicago, IL.

Shicber, Stuart. 1986. An Introduction to Unification Based Approaches to Grammar : CSLI Lecture Notes No. 4, Stanford, CA.

Watt, David. 1980. "Rule Splitting and Attribute Directed Parsing." In N.D.Jones, cd., Semantics-directed Compiler Generation, LNCS 94. Springer-Verlag, NY. Yellin, Danicl. 1988. "Generalized Attributed Parsing." Manuscript; IBM Rescarch, Yorktown Heights, NY. 